\title{
Indicadores de resultado, 2002 Información para la evaluación de los sistemas de salud
}

$\mathrm{L}$

a Secretaría de Salud estableció, dentro del Programa Nacional de Salud 2001-2006, estrategias y líneas de acción específicas para fortalecer el papel rector que le corresponde. Destacan dentro de ellas las actividades de evaluación, que están dirigidas a medir los avances y logros obtenidos en los objetivos finales del sistema de salud: a) mejorar las condiciones de salud de la población; $b$ ) abatir las desigualdades en salud; c) garantizar un trato adecuado en los servicios, y d) asegurar la justicia en el financiamiento en materia de salud. Además de esto, se da seguimiento a los llamados Indicadores de Resultado (IR), que el Consejo Nacional de Salud eligió para evaluar el desempeño de los sistemas estatales de salud, y se evalúan los programas prioritarios

La elaboración y la difusión de los IR 2002 responde a la necesidad de desagregar la información para realizar comparaciones que permitan observar las diferencias en el desempeño de las entidades federativas y las instituciones.

Los IR 2002 (parte II) que aquí se presentan se vinculan con los atributos de disponibilidad y accesibilidad, calidad técnica e interpersonal, y eficiencia de los sistemas de salud. Para la consulta y el análisis de esta información es necesario tomar en cuenta las siguientes consideraciones técnicas:

1. En los cuadros se presenta una columna de total que informa sobre el resultado obtenido en el ámbito nivel sectorial y columnas por institución específica.

2. Para los indicadores con base poblacional del rubro de disponibilidad y accesibilidad por institución se empleó la población legal o potencial notificada para el año de estudio. Para el resultado sectorial (columna de total) se utilizó la población obtenida a partir de las proyecciones de población 2000-2050 del Consejo Nacional de Población, edición 2002.

3. En los cuadros aparecen las siglas ENCI, las cuales significan que la entidad no está cubierta por la institución de la que se trate, esto debido a que las instituciones públicas de salud no tienen presencia en todas las entidades del país.

4. Sólo en las entidades donde se señala que Pemex subroga ser- vicios aparece como valor el cero (0).

Como se puede observar, existen indicadores dentro del rubro de calidad técnica e interpersonal donde sólo se cuenta con resultados generales por entidad federativa y por institución en el plano nacional, lo cual se debe a que las fuentes de información no disponen de datos por entidad federativa e institución de salud. Conjuntamente con las áreas responsables de esta información se están desarrollando las acciones necesarias para contar con la información completa y que pueda ser incorporada en la próxima emisión de los IR por institución.

Finalmente, la difusión de esta información en espacios como esta publicación, forma parte de las acciones que la Secretaría de Salud lleva a cabo para fortalecer los procesos de rendición de cuentas dentro del sector salud, pero también se espera que constituya una herramienta útil para los ámbitos académicos y de investigación. Por esta razón, la difusión de los IR también se realiza por medio del sitio de evaluación del desempeño de la página WEB, de la Secretaría de Salud. Salud, México. 


\section{Cuadro VI.1 \\ INDICADORES DE RESULTADO, DISPONIBILIDAD Y ACCESIBILIDAD, SEGÚN ENTIDAD FEDERATIVA E INSTITUCIÓN. MÉxICO, 2002}

(24) Médicos generales y familiares

por 1000 habitantes, según institución*

Entidad federativa Total SSA IMSS-Sol IMSS ISSSTE Pemex ${ }^{\ddagger}$ Sedena Secmar Total SSA IMSS-Sol IMSS ISSSTE Pemex ${ }^{\ddagger}$ Sedena Secmar

\begin{tabular}{|c|c|c|c|c|c|c|c|c|c|c|c|c|c|c|c|c|}
\hline$N$ acional & 0.53 & 0.59 & 0.37 & 0.35 & 0.47 & 1.37 & 0.97 & 1.33 & 0.44 & 0.36 & 0.08 & 0.37 & 0.76 & 1.78 & 0.88 & 2.18 \\
\hline guascalientes & 0.56 & 0.60 & $\mathrm{ENCl}$ & 0.32 & 0.46 & 0.00 & 1.67 & $\mathrm{ENCl}$ & 0.71 & 0.74 & $\mathrm{ENCl}$ & 0.36 & 0.79 & 0.00 & NDR & $\mathrm{ENCl}$ \\
\hline Baja California & 0.41 & 0.42 & 0.23 & 0.28 & 0.79 & 0.00 & 1.00 & 1.96 & 0.47 & 0.31 & 0.16 & 0.31 & 1.66 & 0.00 & 1.38 & 1.68 \\
\hline Baja California Sur & 0.86 & 0.60 & $\mathrm{ENCl}$ & 0.54 & 0.66 & 0.00 & 0.53 & 2.13 & 0.89 & 0.48 & $\mathrm{ENCl}$ & 0.46 & 1.38 & 0.00 & 1.19 & 2.98 \\
\hline Campeche & 0.91 & 0.67 & 0.27 & 0.32 & 0.52 & 7.52 & 0.19 & 1.30 & 0.56 & 0.39 & 0.09 & 0.29 & 0.82 & 1.70 & NDR & 2.11 \\
\hline Coahuila & 0.50 & 0.43 & 0.38 & 0.34 & 0.50 & 0.00 & 1.14 & $\mathrm{ENCl}$ & 0.53 & 0.34 & 0.14 & 0.36 & 1.05 & 0.00 & 1.71 & $\mathrm{ENCl}$ \\
\hline Colima & 0.74 & 0.58 & $\mathrm{ENCl}$ & 0.32 & 0.62 & 0.00 & 1.51 & 1.42 & 0.69 & 0.31 & $\mathrm{ENCl}$ & 0.45 & 1.49 & 0.00 & NDR & 1.10 \\
\hline Chiapas & 0.53 & 0.51 & 0.38 & 0.36 & 0.56 & 2.41 & 0.60 & 0.76 & 0.26 & 0.18 & 0.07 & 0.30 & 0.68 & 0.48 & 0.32 & 1.01 \\
\hline Chihuahua & 0.47 & 0.57 & 0.62 & 0.34 & 0.43 & 0.81 & 0.43 & $\mathrm{ENCl}$ & 0.38 & 0.43 & 0.15 & 0.26 & 0.94 & NDR & 0.48 & $\mathrm{ENCl}$ \\
\hline Distrito Federal & 0.67 & 0.54 & 0.08 & 0.31 & 0.39 & 0.99 & 1.72 & 0.51 & 1.24 & 1.12 & NDR & 0.54 & 0.67 & 4.35 & 1.36 & 3.54 \\
\hline Durango & 0.73 & 0.61 & 0.60 & 0.46 & 0.42 & 0.00 & 1.29 & $\mathrm{ENCl}$ & 0.49 & 0.33 & 0.14 & 0.38 & 0.64 & 0.00 & 0.48 & $\mathrm{ENCl}$ \\
\hline Guanajuato & 0.43 & 0.65 & $\mathrm{ENCl}$ & 0.30 & 0.44 & 0.99 & 0.30 & $\mathrm{ENCl}$ & 0.27 & 0.20 & $\mathrm{ENCl}$ & 0.27 & 0.81 & 2.27 & 0.25 & $\mathrm{ENCl}$ \\
\hline Guerrero & 0.67 & 0.61 & $\mathrm{ENCl}$ & 0.43 & 0.43 & 0.00 & 0.55 & 3.64 & 0.29 & 0.17 & $\mathrm{ENCl}$ & 0.35 & 0.53 & 0.00 & 0.31 & 3.89 \\
\hline Hidalgo & 0.62 & 0.70 & 0.35 & 0.30 & 0.38 & 1.77 & 0.36 & $\mathrm{ENCl}$ & 0.32 & 0.20 & 0.08 & 0.38 & 0.68 & 2.05 & NDR & $\mathrm{ENCl}$ \\
\hline Jalisco & 0.51 & 0.56 & $\mathrm{ENCl}$ & 0.38 & 0.45 & 2.59 & 1.67 & 0.90 & 0.44 & 0.35 & $\mathrm{ENCl}$ & 0.36 & 0.92 & 0.86 & 3.82 & 3.14 \\
\hline México & 0.35 & 0.49 & $\mathrm{ENCl}$ & 0.48 & 0.33 & 0.73 & 0.67 & $\mathrm{ENCl}$ & 0.24 & 0.23 & $\mathrm{ENCl}$ & 0.41 & 0.13 & 0.26 & 0.61 & $\mathrm{ENCl}$ \\
\hline Michoacán & 0.47 & 0.66 & 0.31 & 0.27 & 0.66 & 0.00 & 0.90 & 1.10 & 0.32 & 0.34 & 0.06 & 0.32 & 1.01 & 0.00 & 0.60 & 1.00 \\
\hline Morelos & 0.45 & 0.54 & $\mathrm{ENCl}$ & 0.32 & 0.51 & 0.00 & 0.61 & $\mathrm{ENCl}$ & 0.46 & 0.36 & $\mathrm{ENCl}$ & 0.48 & 0.93 & 0.00 & 0.76 & $\mathrm{ENCl}$ \\
\hline $\mathrm{N}$ ayarit & 0.71 & 0.63 & 0.62 & 0.32 & 0.57 & 0.00 & 0.73 & 2.18 & 0.49 & 0.29 & 0.08 & 0.43 & 0.85 & 0.00 & NDR & NDR \\
\hline N uevo León & 0.44 & 0.39 & $\mathrm{ENCl}$ & 0.28 & 0.67 & 1.23 & 0.51 & $\mathrm{ENCl}$ & 0.39 & 0.17 & $\mathrm{ENCl}$ & 0.34 & 0.95 & 1.96 & 0.68 & $\mathrm{ENCl}$ \\
\hline 0 axaca & 0.55 & 0.61 & 0.39 & 0.30 & 0.48 & 1.47 & 1.12 & 1.40 & 0.25 & 0.18 & 0.08 & 0.25 & 0.74 & 1.47 & 0.80 & 1.90 \\
\hline Puebla & 0.44 & 0.65 & 0.28 & 0.31 & 0.54 & 1.80 & 0.34 & $\mathrm{ENCl}$ & 0.27 & 0.18 & 0.06 & 0.30 & 0.69 & 0.72 & 0.34 & $\mathrm{ENCl}$ \\
\hline Q uerétaro & 0.53 & 0.62 & $\mathrm{ENCl}$ & 0.25 & 0.47 & 0.00 & 0.30 & $\mathrm{ENCl}$ & 0.41 & 0.37 & $\mathrm{ENCl}$ & 0.22 & 0.97 & 0.00 & NDR & $\mathrm{ENCl}$ \\
\hline Q uintana Roo & 0.60 & 0.56 & $\mathrm{ENCl}$ & 0.28 & 0.49 & $\mathrm{ENCl}$ & 0.57 & 1.58 & 0.46 & 0.27 & $\mathrm{ENCl}$ & 0.32 & 0.98 & $\mathrm{ENCl}$ & 0.45 & 1.13 \\
\hline San Luis Potosí & 0.48 & 0.49 & 0.34 & 0.31 & 0.41 & 2.29 & 0.35 & $\mathrm{ENCl}$ & 0.33 & 0.33 & 0.08 & 0.22 & 0.73 & 1.76 & 0.69 & $\mathrm{ENCl}$ \\
\hline Sinaloa & 0.58 & 0.75 & 0.41 & 0.48 & 0.57 & 0.57 & 0.66 & 4.74 & 0.43 & 0.51 & 0.11 & 0.33 & 0.83 & 0.28 & 0.56 & 6.32 \\
\hline Sonora & 0.54 & 0.62 & $\mathrm{ENCl}$ & 0.41 & 0.54 & 0.00 & 0.89 & 4.16 & 0.62 & 0.57 & $\mathrm{ENCl}$ & 0.48 & 1.22 & 0.00 & 0.40 & 4.16 \\
\hline Tabasco & 0.82 & 0.66 & $\mathrm{ENCl}$ & 0.31 & 0.48 & 1.30 & 0.76 & 0.88 & 0.46 & 0.27 & $\mathrm{ENCl}$ & 0.27 & 0.69 & 1.63 & 0.86 & 1.10 \\
\hline Tamaulipas & 0.61 & 0.75 & 0.41 & 0.35 & 0.53 & 1.04 & 0.74 & 1.79 & 0.60 & 0.58 & 0.09 & 0.31 & 1.18 & 2.04 & 0.62 & 0.83 \\
\hline Tlaxcala & 0.70 & 0.87 & $\mathrm{ENCl}$ & 0.40 & 0.32 & 0.00 & 0.51 & $\mathrm{ENCl}$ & 0.34 & 0.27 & $\mathrm{ENCl}$ & 0.38 & 0.56 & 0.00 & NDR & $\mathrm{ENCl}$ \\
\hline Veracruz & 0.58 & 0.76 & 0.39 & 0.41 & 0.68 & 1.22 & 0.56 & 0.99 & 0.36 & 0.30 & 0.05 & 0.39 & 0.84 & 1.35 & 0.44 & 1.65 \\
\hline Yucatán & 0.49 & 0.64 & 0.31 & 0.25 & 0.73 & 0.00 & 0.20 & 1.33 & 0.47 & 0.40 & 0.12 & 0.42 & 1.04 & 0.00 & 0.27 & 3.56 \\
\hline & 067 & 50 & 044 & 40 & 062 & 000 & 0.52 & ENCl & 038 & 079 & 0.14 & 0.20 & 0.98 & 0.00 & 0.78 & ENC \\
\hline
\end{tabular}

* El denominador para el total se basa en las proyecciones de población 2000-2050 del Consejo N acional de Población, 2002. Para los indicadores por institución se utilizó la población legal o potencial reportada en el Boletín de Información Estadística 2002, Dirección General de Información y Evaluación del Desempeño (D GIED), Secretaría de Salud, México. El numerador se obtuvo del Boletín de Información Estadística 2002, D G IED

* Petróleos Mexicanos subroga servicios en las entidades de Aguascalientes, Baja California, Baja California Sur, Coahuila, Colima, D urango, Guerrero, Michoacán, Morelos, N ayarit, Q uerétaro, Sonora,Tlaxcala,Yucatán y Zacatecas

EN Cl: entidad no cubierta por la institución

NDR: no dispone de este tipo de recursos

Fuente: Boletín de Información Estadística 2002;1(22). Recursos y Servicios. Dirección General de Información y Evaluación del Desempeño, Secretaría de Salud, y proyecciones de población del Consejo N acional de Población, México, 2002 


\section{Cuadro VI.2 \\ INDICADORES DE RESULTADO, DISPONIBILIDAD Y ACCESIBILIDAD, SEGÚN ENTIDAD FEDERATIVA E INSTITUCIÓN. MÉxICO, 2002}

(26) Camas censables*

por 1000 habitantes, según institución ${ }^{\ddagger}$

Entidad federativa Total SSA IMSS-Sol IMSS ISSSTE Pemex ${ }^{5}$ Sedena Secmar
(27-28) Hospitales, según número de camas como porcentaje del total de hospitales por entidad federativa Hospitales con menos de 30 camas Hospitales con 30 camas y más

\begin{tabular}{|c|c|c|c|c|c|c|c|c|c|c|}
\hline$N$ acional & 0.72 & 0.69 & 0.21 & 0.62 & 0.66 & 1.42 & 5.21 & 3.79 & 47.2 & 52.8 \\
\hline Aguascalientes & 0.78 & 0.81 & $\mathrm{ENCl}$ & 0.47 & 0.78 & 0.00 & NDR & $\mathrm{ENCl}$ & 40.0 & 60.0 \\
\hline Baja California & 0.60 & 0.44 & 0.29 & 0.52 & 1.06 & 0.00 & 5.44 & 6.71 & 43.3 & 56.7 \\
\hline Baja California Sur & 1.09 & 0.65 & $\mathrm{ENCl}$ & 0.59 & 0.87 & 0.00 & 5.96 & 5.96 & 77.8 & 22.2 \\
\hline Campeche & 0.88 & 0.78 & 0.23 & 0.42 & 0.60 & 1.16 & NDR & 4.51 & 66.7 & 33.3 \\
\hline Coahuila & 0.89 & 0.51 & 0.33 & 0.73 & 0.86 & 0.00 & 9.48 & $\mathrm{ENCl}$ & 41.7 & 58.3 \\
\hline Colima & 0.86 & 0.56 & $\mathrm{ENCl}$ & 0.46 & 0.93 & 0.00 & NDR & 2.33 & 20.0 & 80.0 \\
\hline Chiapas & 0.36 & 0.32 & 0.18 & 0.37 & 0.68 & NDR & 1.90 & 3.16 & 61.9 & 38.1 \\
\hline Chihuahua & 0.73 & 0.98 & 0.32 & 0.52 & 0.84 & NDR & 4.04 & $\mathrm{ENCl}$ & 57.4 & 42.6 \\
\hline Distrito Federal & 1.88 & 1.73 & NDR & 0.87 & 0.66 & 3.97 & 8.15 & 2.92 & 15.7 & 84.3 \\
\hline Durango & 0.81 & 0.66 & 0.30 & 0.60 & 0.59 & 0.00 & 5.31 & $\mathrm{ENCl}$ & 47.8 & 52.2 \\
\hline Guanajuato & 0.48 & 0.47 & $\mathrm{ENCl}$ & 0.50 & 0.63 & 1.45 & 5.02 & $\mathrm{ENCl}$ & 48.6 & 51.4 \\
\hline Guerrero & 0.41 & 0.24 & $\mathrm{ENCl}$ & 0.53 & 0.39 & 0.00 & 3.62 & 8.15 & 48.1 & 51.9 \\
\hline Hidalgo & 0.50 & 0.45 & 0.29 & 0.39 & 0.59 & 0.67 & NDR & $\mathrm{ENCl}$ & 57.1 & 42.9 \\
\hline Jalisco & 0.89 & 0.79 & $\mathrm{ENCl}$ & 0.76 & 0.85 & NDR & 7.01 & 15.25 & 47.4 & 52.6 \\
\hline México & 0.48 & 0.81 & $\mathrm{ENCl}$ & 0.52 & 0.12 & NDR & 3.00 & $\mathrm{ENCl}$ & 29.7 & 70.3 \\
\hline Michoacán & 0.49 & 0.62 & 0.14 & 0.51 & 0.82 & 0.00 & 3.39 & 1.10 & 52.0 & 48.0 \\
\hline Morelos & 0.50 & 0.39 & $\mathrm{ENCl}$ & 0.54 & 0.72 & 0.00 & 6.05 & $\mathrm{ENCl}$ & 16.7 & 83.3 \\
\hline $\bar{N}$ ayarit & 0.57 & 0.33 & 0.24 & 0.55 & 0.71 & 0.00 & NDR & 5.46 & 50.0 & 50.0 \\
\hline N uevo León & 0.78 & 0.31 & $\mathrm{ENCl}$ & 0.75 & 0.93 & 1.09 & 9.13 & $\mathrm{ENCl}$ & 44.8 & 55.2 \\
\hline 0 axaca & 0.47 & 0.48 & 0.20 & 0.35 & 0.60 & 1.23 & 7.28 & 6.11 & 45.0 & 55.0 \\
\hline Puebla & 0.61 & 0.72 & 0.20 & 0.59 & 0.67 & 0.29 & 3.42 & $\mathrm{ENCl}$ & 66.1 & 33.9 \\
\hline Q uerétaro & 0.48 & 0.43 & $\mathrm{ENCl}$ & 0.31 & 0.64 & 0.00 & NDR & $\mathrm{ENCl}$ & 11.1 & 88.9 \\
\hline Q uintana Roo & 0.51 & 0.33 & $\mathrm{ENCl}$ & 0.32 & 0.54 & $\mathrm{ENCl}$ & 3.41 & 3.38 & 53.3 & 46.7 \\
\hline San Luis Potosí & 0.57 & 0.58 & 0.22 & 0.43 & 0.59 & 0.70 & 8.98 & $\mathrm{ENCl}$ & 43.5 & 56.5 \\
\hline Sinaloa & 0.67 & 0.71 & 0.19 & 0.64 & 0.78 & NDR & 4.71 & 11.51 & 63.6 & 36.4 \\
\hline Sonora & 0.96 & 1.20 & $\mathrm{ENCl}$ & 0.71 & 0.88 & 0.00 & 2.97 & 7.03 & 57.8 & 42.2 \\
\hline Tabasco & 0.64 & 0.47 & $\mathrm{ENCl}$ & 0.33 & 0.45 & 1.22 & 3.61 & 4.63 & 60.0 & 40.0 \\
\hline Tamaulipas & 0.86 & 0.98 & 0.16 & 0.47 & 1.18 & 1.87 & 6.18 & 2.90 & 25.0 & 75.0 \\
\hline Tlaxcala & 0.48 & 0.40 & $\mathrm{ENCl}$ & 0.49 & 0.76 & 0.00 & 0.00 & $\mathrm{ENCl}$ & 22.2 & 77.8 \\
\hline Veracruz & 0.59 & 0.65 & 0.13 & 0.66 & 0.61 & 1.13 & 2.54 & 2.64 & 59.8 & 40.2 \\
\hline Yucatán & 0.84 & 0.80 & 0.27 & 0.75 & 0.78 & 0.00 & 4.03 & 4.45 & 52.0 & 48.0 \\
\hline Zacatecas & 0.47 & 0.31 & 0.33 & 0.39 & 0.64 & 0.00 & 7.77 & $\mathrm{ENCl}$ & 50.0 & 50.0 \\
\hline
\end{tabular}

* Sólo incluye las camas censables de unidades hospitalarias generales y de especialidad

₹ El denominador para el total se basa en las proyecciones de población 2000-2050 del Consejo N acional de Población, 2002. Para los indicadores por institución se utilizó la población legal o potencial reportada en el Boletín de Información Estadística 2002, Dirección General de Información y Evaluación del Desempeño (DGIED), Secretaría de Salud, México. El numerador se obtuvo del Boletín de Información Estadística 2002, D G IED

$\S$ Petróleos Mexicanos subroga servicios en las entidades de A guascalientes, Baja California, Baja California Sur, Coahuila, Colima, Durango, Guerrero, Michoacán, Morelos, N ayarit, Q uerétaro, Sonora,T laxcala,Yucatán y Zacatecas

EN Cl: entidad no cubierta por la institución NDR: no dispone de este tipo de recursos

Fuentes: Boletín de Información Estadística 2002;1(22). Recursos y Servicios, Dirección General de Información y Evaluación del D esempeño, Secretaría de Salud, y proyecciones de población del Consejo N acional de Población, 2002, México 


\section{Cuadro VI.3 \\ INDICADORES DE RESULTADO, DISPONIBILIDAD Y ACCESIBILIDAD, SEGÚN INSTITUCIÓN. MÉxıCO, 2002}

\begin{tabular}{|c|c|c|c|c|c|c|c|c|c|}
\hline \multirow{3}{*}{$\begin{array}{l}\text { Nivel } \\
\text { Nacional }\end{array}$} & \multicolumn{9}{|c|}{ mas como porcentaje del total de hospitales por } \\
\hline & Total no asegurados & SSA & IMSS-Sol & Total asegurados & IMSS & ISSSTE & Pemex* & Sedena & Secmar \\
\hline & 49.1 & 51.5 & 34.8 & 45.4 & 47.3 & 39.4 & 60.9 & 19.0 & 71.9 \\
\hline & \multicolumn{9}{|c|}{ (28) Hospitales de 30 y más camas como porcentaje del total de hospitales por institución a nivel nacional } \\
\hline$N$ acional & 50.9 & 48.5 & 65.2 & 54.6 & 52.7 & 60.6 & 39.1 & 81.0 & 28.1 \\
\hline
\end{tabular}




\section{Cuadro VI.4 \\ INDICADORES DE RESULTADO, DISPONIBILIDAD Y ACCESIBILIDAD, SEGÚN ENTIDAD FEDERATIVA E INSTITUCIÓN. MÉxICO, 2002}

(29) Usuarias activas de métodos de PF por 100 mujeres en edad fértil*

(30) Razón de consultas prenatales por embarazada

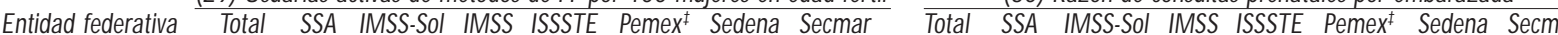

\begin{tabular}{|c|c|c|c|c|c|c|c|c|c|c|c|c|c|c|c|c|}
\hline$N$ acional & 35.82 & 23.43 & 49.61 & 45.93 & 37.21 & 73.90 & 99.85 & 5.69 & 4.59 & 3.59 & 7.43 & 6.38 & 2.90 & 4.96 & 4.02 & 4.0 \\
\hline Aguascalientes & 35.01 & 21.18 & $\mathrm{ENCl}$ & 49.89 & 37.40 & 0.00 & 300.97 & $\mathrm{ENCl}$ & 4.47 & 3.42 & $\mathrm{ENCl}$ & 6.28 & 1.62 & 0.00 & NRA & $\mathrm{ENCl}$ \\
\hline Baja California & 37.21 & 13.57 & 40.50 & 50.83 & 43.29 & 0.00 & 150.02 & 10.97 & 4.98 & 3.42 & 5.31 & 6.18 & 3.22 & 0.00 & 3.70 & 5.15 \\
\hline Baja California Sur & 45.41 & 18.90 & $\mathrm{ENCl}$ & 161.61 & 35.07 & 0.00 & 298.27 & 7.78 & 4.95 & 4.37 & $\mathrm{ENCl}$ & 5.79 & 4.28 & 0.00 & 2.77 & 60 \\
\hline Campeche & 45.08 & 3.72 & 42.71 & 286.86 & 29.11 & 111.89 & 184.99 & 8.94 & 5.66 & 4.62 & 8.83 & 7.70 & 3.59 & 3.27 & NRA & .05 \\
\hline Coahuila & 41.74 & 1.14 & 49.35 & 55.02 & 37.69 & 0.00 & 193.25 & $\mathrm{ENCl}$ & 4.58 & 2.86 & 7.81 & 5.64 & 4.54 & 0.00 & 5.73 & $\mathrm{NCl}$ \\
\hline Colima & 48.63 & 23.55 & $\mathrm{ENCl}$ & 240.27 & 42.82 & 0.00 & 626.69 & 8.42 & 5.09 & 3.67 & $\mathrm{ENCl}$ & 7.49 & 10.01 & 0.00 & NRA & 2.92 \\
\hline Chiapas & 38.54 & 25.51 & 54.75 & 51.84 & 29.71 & 7.00 & 19.58 & 0.90 & 4.85 & 3.50 & 7.77 & 6.05 & 2.19 & 4.11 & 4.23 & 3.59 \\
\hline Chihuahua & 37.07 & 17.90 & 58.05 & 44.05 & 36.72 & 2.93 & 105.67 & $\mathrm{ENCl}$ & 5.14 & 3.21 & 5.78 & 6.44 & 3.09 & 8.14 & 2.94 & $\mathrm{NCl}$ \\
\hline Distrito Federal & 40.23 & 7.65 & 25.94 & 112.41 & 36.34 & 58.10 & 55.47 & 2.63 & 3.93 & 2.83 & 7.36 & 6.88 & 2.50 & 6.03 & 3.59 & 2.61 \\
\hline urango & 43.60 & 21.05 & 55.60 & 135.45 & 29.80 & 0.00 & 215.42 & $\mathrm{ENCl}$ & 4.41 & 3.37 & 8.08 & 5.49 & 2.70 & 0.00 & 3.87 & $\mathrm{ENCl}$ \\
\hline uanajuato & 24.37 & 17.24 & $\mathrm{ENCl}$ & 26.56 & 47.34 & 28.11 & 33.99 & $\mathrm{ENCl}$ & 4.49 & 3.56 & $\mathrm{ENCl}$ & 6.59 & 3.40 & 1.83 & 4.49 & $\mathrm{NCl}$ \\
\hline Guerrero & 38.70 & 28.07 & $\mathrm{ENCl}$ & 560.40 & 35.94 & 0.00 & 151.58 & 14.03 & 3.90 & 3.52 & $\mathrm{ENCl}$ & 7.70 & 2.66 & 0.00 & 3.31 & 3.09 \\
\hline Hidalgo & 45.20 & 27.23 & 51.69 & 131.54 & 43.10 & 133.32 & 171.43 & $\mathrm{ENCl}$ & 4.51 & 3.78 & 6.46 & 6.53 & 2.60 & 8.28 & NRA & $\mathrm{ENCl}$ \\
\hline Jalisco & 27.06 & 19.32 & $\mathrm{ENCl}$ & 32.99 & 35.00 & 54.23 & 129.11 & 11.39 & 5.01 & 4.28 & $\mathrm{ENCl}$ & 5.97 & 3.49 & 4.67 & 4.41 & 8.53 \\
\hline éxico & 29.14 & 35.62 & $\mathrm{ENCl}$ & 24.68 & 23.19 & 12.02 & 62.02 & $\mathrm{ENCl}$ & 3.76 & 3.00 & $\mathrm{ENCl}$ & 6.22 & 2.89 & 2.82 & 4.28 & $\mathrm{ENCl}$ \\
\hline Michoacán & 33.69 & 24.88 & 39.16 & 36.59 & 46.52 & 0.00 & 58.26 & 5.23 & 4.40 & 3.35 & 7.18 & 6.24 & 3.20 & 0.00 & 7.08 & 4.82 \\
\hline Morelos & 32.78 & 24.81 & $\mathrm{ENCl}$ & 38.92 & 48.26 & 0.00 & 147.79 & $\mathrm{ENCl}$ & 4.31 & 3.78 & $\mathrm{ENCl}$ & 5.52 & 3.07 & 0.00 & 3.66 & $\mathrm{ENCl}$ \\
\hline ayarit & 45.11 & 22.11 & 45.06 & 241.20 & 34.70 & 0.00 & 387.50 & 10.55 & 4.80 & 4.34 & 6.55 & 6.19 & 2.47 & 0.00 & NRA & \\
\hline uevo León & 36.02 & 15.19 & $\mathrm{ENCl}$ & 50.76 & 88.27 & 164.04 & 224.01 & $\mathrm{ENCl}$ & 5.70 & 4.39 & $\mathrm{ENCl}$ & 6.83 & 3.54 & 7.61 & 5.09 & $\mathrm{ENCl}$ \\
\hline 0 axaca & 38.44 & 26.66 & 46.02 & 71.96 & 27.72 & 150.04 & 198.97 & 1.86 & 4.99 & 4.09 & 6.95 & 6.24 & 3.24 & 4.86 & 4.28 & 6.21 \\
\hline Puebla & 35.04 & 32.78 & 50.97 & 27.46 & 36.41 & 155.22 & 79.75 & $\mathrm{ENCl}$ & 4.66 & 3.58 & 7.80 & 6.21 & 2.77 & 8.05 & 4.54 & $\mathrm{ENCl}$ \\
\hline Q uerétaro & 34.95 & 17.40 & $\mathrm{ENCl}$ & 59.14 & 43.68 & 0.00 & NRA & $\mathrm{ENCl}$ & 5.39 & 3.84 & $\mathrm{ENCl}$ & 8.33 & 2.20 & 0.00 & NRA & $\mathrm{ENCl}$ \\
\hline Q uintana Roo & 32.34 & 14.14 & $\mathrm{ENCl}$ & 85.02 & 33.97 & $\mathrm{ENCl}$ & 171.37 & 0.28 & 4.54 & 3.31 & $\mathrm{ENCl}$ & 6.21 & 3.93 & $\mathrm{ENCl}$ & 13.26 & 8.97 \\
\hline San Luis Potosí & 36.45 & 21.66 & 49.61 & 48.59 & 28.95 & 98.31 & 229.19 & $\mathrm{ENCl}$ & 4.95 & 4.13 & 6.87 & 5.66 & 2.78 & 7.37 & 5.23 & $\mathrm{ENCl}$ \\
\hline Sinaloa & 41.27 & 18.39 & 48.83 & 51.24 & 49.29 & 4.43 & 64.93 & NRA & 4.38 & 3.09 & 6.93 & 5.58 & 2.58 & 12.55 & 7.07 & 6.48 \\
\hline Sonora & 34.69 & 21.54 & $\mathrm{ENCl}$ & 42.61 & 45.93 & 0.00 & 288.41 & 4.33 & 4.49 & 3.51 & $\mathrm{ENCl}$ & 5.82 & 3.58 & 0.00 & 4.20 & 4.76 \\
\hline Tabasco & 42.84 & 29.01 & $\mathrm{ENCl}$ & 250.03 & 21.33 & 77.28 & 65.83 & 15.69 & 3.98 & 4.36 & $\mathrm{ENCl}$ & 6.11 & 2.56 & 6.66 & 3.00 & 3.08 \\
\hline Tamaulipas & 34.21 & 15.37 & 47.24 & 44.34 & 38.57 & 69.71 & 97.74 & 5.52 & 5.41 & 3.71 & 6.90 & 7.65 & 3.71 & 4.73 & 3.81 & 3.39 \\
\hline Tlaxcala & 35.33 & 31.05 & $\mathrm{ENCl}$ & 45.14 & 29.46 & 0.00 & 93.29 & $\mathrm{ENCl}$ & 4.23 & 3.51 & $\mathrm{ENCl}$ & 6.61 & 4.14 & 0.00 & NRA & $\mathrm{ENCl}$ \\
\hline Veracruz & 42.58 & 33.12 & 55.84 & 43.96 & 39.44 & 69.26 & 278.69 & 7.34 & 5.28 & 4.23 & 7.95 & 6.93 & 2.59 & 4.89 & 4.71 & 4.31 \\
\hline Yucatán & 36.23 & 29.95 & 38.45 & 44.24 & 25.42 & 0.00 & 51.83 & 4.51 & 4.94 & 3.36 & 8.82 & 6.07 & 5.62 & 0.00 & 5.40 & 4.50 \\
\hline acatecas & 43.68 & 28.92 & 53.98 & 71.00 & 48.75 & 0.00 & 103.36 & $\mathrm{ENCl}$ & 5.03 & 4.03 & 7.86 & 6.23 & 3.09 & 0.00 & 9.03 & \\
\hline
\end{tabular}

* El denominador (mujeres en edad fértil) para el total se basa en las proyecciones de población 2000-2050 del Consejo N acional de Población, 2002. Para los indicadores por institución se utilizó mujeres en edad fértil de la población usuaria por institución reportada en el Boletín de Información Estadística 2002, Dirección General de Información y Evaluación del Desempeño (D G IED), Secretaría de Salud, México. El numerador se obtuvo del Boletín de Información Estadística 2002, D GIED

₹ Petróleos Mexicanos subroga servicios en las entidades de Aguascalientes, Baja California, Baja California Sur, Coahuila, Colima, Durango, Guerrero, Michoacán, Morelos, N ayarit, Q uerétaro, Sonora,Tlaxcala, Yucatán y Zacatecas

EN Cl: entidad no cubierta por la institución

NRA: no reporta acciones para este indicador

Fuentes: Boletín de Información Estadística 2002;1(22). Recursos y Servicios, Dirección General de Información y Evaluación del D esempeño, Secretaría de Salud, y proyecciones de población del Consejo N acional de Población, México, 2002 


\section{Cuadro VI.5 \\ INDICADORES DE RESULTADO, DISPONIBILIDAD Y ACCESIBILIDAD, SEGÚN ENTIDAD FEDERATIVA E INSTITUCIÓN. MÉxico, 2002}

(35) Consultas de medicina de especialidad por 1000 habitantes*

Entidad federativa Total SSA IMSS-Sol IMSS ISSSTE Pemex ${ }^{\ddagger}$ Sedena Secmar

$\begin{array}{llllllllll}365.7 & 213.8 & 35.2 & 360.2 & 592.4 & 2814.3 & 1802.5 & 1766.8\end{array}$

Nacional

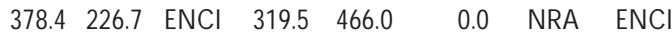

Aguascalientes

$\begin{array}{lllll}341.5 & 172.2 & 56.8 & 244.5 & 973.2\end{array}$

0.01328 .12463 .4

Baja California

Baja California Sur 534

Coahuila

$\begin{array}{lllll}54.7 & 149.1 & \mathrm{ENCl} & 447.1 & 709.1\end{array}$

0.01878 .81418 .4

Colima

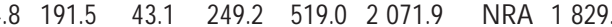

Chiapas $\begin{array}{llllllll}449.3 & 214.7 & 65.2 & 367.2 & 819.7 & 0.0 & 2232.2 & \mathrm{ENCl}\end{array}$

Chihuahua

$\begin{array}{llllllll}499.6 & 165.9 & \mathrm{ENCl} & 432.7 & 831.9 & 0.0 & \text { NRA } & 1395.8\end{array}$

(36) Intervenciones quirúrgicas por 1000 habitantes* Total SSA IMSS-Sol IMSS ISSSTE Pemex ${ }^{\ddagger}$ Sedena Secmar
Distrit

Durango

Guanajuato

Guerrero

Hidalgo

Jalisco

México

Michoacán

Morelos

$\mathrm{N}$ ayarit

N uevo León

0 axaca

Puebla

Q uerétaro

Q uintana Roo

San Luis Potos

Sinaloa

Sonora

Tabasco

Tamaulipas

Tlaxcala

Veracruz

Yucatán

Zacatecas

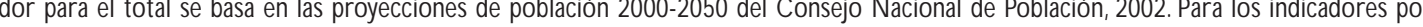
institución se utilizó la población legal o potencial obtenida del Boletín de Información Estadística 2002, Dirección General de Información y Evaluación del Desempeño, Secretaría de Salud, México

₹ Petróleos Mexicanos subroga servicios en las entidades de Aguascalientes, Baja California, Baja California Sur, Coahuila, Colima, Durango, Guerrero, Michoacán, Morelos, N ayarit, Q uerétaro, Sonora, Tlaxcala, Yucatán y Zacatecas

EN Cl: entidad no cubierta por la institución

NRA: no reporta acciones para este indicador

Fuente: Bo letín de Información Estadística 2002;1(22). Recursos y Servicios. Dirección General de Información y Evaluación del D esempeño, Secretaría de Salud, y proyecciones de población del Consejo Nacional de Población, México, 2002 


\section{Cuadro VII.1 \\ INDICADORES DE RESULTADO, CALIDAD TÉCNICA E INTERPERSONAL, SEGÚN ENTIDAD FEDERATIVA E INSTITUCIÓN. MÉxICO, 2002}

Calidad técnica e interpersonal

\begin{tabular}{|c|c|c|c|c|c|c|c|c|c|c|c|c|c|c|}
\hline \multirow[b]{3}{*}{ Entidad Federativa } & \multicolumn{14}{|c|}{ Calidad técnica e interpersonal } \\
\hline & \multicolumn{9}{|c|}{ (39) Porcentaje de nacimientos por cesárea* } & \multicolumn{5}{|c|}{$\begin{array}{c}\text { (40) \% de recetas surtidas en forma } \\
\text { completa en el primer nivel de atención } \\
\text { nacional por institución } \neq\end{array}$} \\
\hline & Total & SSA & IMSS-SOl & IMSS & ISSSTE & Pemex ${ }^{\ddagger}$ & Sedena & Secmar & Privada & Total & SSA & IMSS & ISSSTE & Otras \\
\hline Nacional & 39.06 & 27.76 & 17.14 & 40.18 & 56.78 & 42.07 & 27.60 & 48.08 & 58.93 & 91.81 & 81.06 & 92.06 & 92.74 & 95.29 \\
\hline Aguascalientes & 39.35 & 38.12 & $\mathrm{ENCl}$ & 32.20 & 41.64 & 0.00 & NRA & $\mathrm{ENCl}$ & 59.53 & 82.51 & & & & \\
\hline Baja California & 38.92 & 23.60 & 27.45 & 39.52 & 57.12 & 0.00 & 23.36 & 64.06 & 54.10 & NRD & & & & \\
\hline Baja California Sur & 42.34 & 33.60 & $\mathrm{ENCl}$ & 45.38 & 54.75 & 0.00 & 29.73 & 53.57 & 68.27 & 95.69 & & & & \\
\hline Campeche & 36.83 & 31.99 & 16.62 & 40.82 & 58.58 & 57.44 & NRA & 43.09 & 71.39 & 90.63 & & & & \\
\hline Coahuila & 37.25 & 27.39 & 16.11 & 36.05 & 50.21 & 0.00 & 33.06 & $\mathrm{ENCl}$ & 63.34 & 90.71 & & & & \\
\hline Colima & 40.34 & 30.88 & $\mathrm{ENCl}$ & 41.89 & 63.84 & 0.00 & NRA & 34.62 & 65.15 & 92.13 & & & & \\
\hline Chiapas & 31.03 & 30.24 & 12.58 & 45.73 & 53.69 & NRA & 24.77 & 36.89 & 56.60 & 90.89 & & & & \\
\hline Chihuahua & 36.48 & 30.11 & 21.58 & 31.62 & 57.62 & NRA & 4.55 & $\mathrm{ENCl}$ & 54.39 & 94.83 & & & & \\
\hline Distrito Federal & 45.13 & 31.51 & NRA & 45.87 & 55.27 & 45.82 & 28.37 & 64.15 & 63.71 & $N P$ & & & & \\
\hline Durango & 30.86 & 24.63 & 18.64 & 33.27 & 50.06 & 0.00 & 22.29 & $\mathrm{ENCl}$ & 46.92 & 88.77 & & & & \\
\hline Guanajuato & 37.17 & 24.00 & $\mathrm{ENCl}$ & 39.55 & 62.36 & 46.55 & 15.74 & $\mathrm{ENCl}$ & 48.76 & 92.92 & & & & \\
\hline Guerrero & 33.01 & 23.99 & $\mathrm{ENCl}$ & 35.27 & 53.08 & 0.00 & 40.10 & 41.48 & 51.64 & 97.02 & & & & \\
\hline Hidalgo & 35.02 & 29.67 & 16.93 & 44.22 & 55.51 & 54.74 & NRA & $\mathrm{ENCl}$ & 52.51 & 96.12 & & & & \\
\hline Jalisco & 40.75 & 23.08 & $\mathrm{ENCl}$ & 42.14 & 53.69 & NRA & 22.01 & 39.53 & 57.15 & 81.30 & & & & \\
\hline México & 39.06 & 22.05 & $\mathrm{ENCl}$ & 36.31 & 56.76 & NRA & 13.09 & $\mathrm{ENCl}$ & 56.73 & 94.74 & & & & \\
\hline Michoacán & 37.01 & 27.11 & 18.25 & 43.87 & 55.86 & 0.00 & 21.21 & 35.16 & 49.99 & 78.06 & & & & \\
\hline Morelos & 41.69 & 28.57 & $\mathrm{ENCl}$ & 44.39 & 57.54 & 0.00 & 38.61 & $\mathrm{ENCl}$ & 61.29 & 91.77 & & & & \\
\hline$N$ ayarit & 32.43 & 22.79 & 20.34 & 40.45 & 49.03 & 0.00 & NRA & NRA & 53.48 & 95.70 & & & & \\
\hline N uevo León & 50.28 & 32.15 & $\mathrm{ENCl}$ & 43.34 & 69.52 & 34.69 & 5.00 & $\mathrm{ENCl}$ & 72.55 & 89.96 & & & & \\
\hline 0 axaca & 34.89 & 30.65 & 18.04 & 49.19 & 59.79 & 47.46 & 35.77 & 52.26 & 58.00 & 95.71 & & & & \\
\hline Puebla & 39.86 & 19.72 & 18.88 & 43.00 & 64.44 & 5.33 & 37.92 & $\mathrm{ENCl}$ & 69.56 & 91.87 & & & & \\
\hline Q uerétaro & 39.28 & 30.33 & $\mathrm{ENCl}$ & 36.48 & 60.72 & 0.00 & NRA & $\mathrm{ENCl}$ & 63.17 & 96.31 & & & & \\
\hline Q uintana Roo & 37.70 & 26.66 & $\mathrm{ENCl}$ & 40.44 & 56.81 & $\mathrm{ENCl}$ & 26.32 & 37.19 & 56.49 & 97.41 & & & & \\
\hline San Luis Potosí & 28.70 & 24.55 & 14.99 & 29.89 & 43.59 & 25.71 & 33.33 & $\mathrm{ENCl}$ & 46.78 & 90.99 & & & & \\
\hline Sinaloa & 42.00 & 34.05 & 17.99 & 40.01 & 60.18 & NRA & 44.35 & 46.53 & 62.90 & 88.93 & & & & \\
\hline Sonora & 38.30 & 26.24 & $\mathrm{ENCl}$ & 40.47 & 58.36 & 0.00 & 29.11 & 47.73 & 58.18 & 91.61 & & & & \\
\hline Tabasco & 40.42 & 33.32 & $\mathrm{ENCl}$ & 42.45 & 73.03 & 57.30 & 29.83 & 19.72 & 65.94 & 91.96 & & & & \\
\hline Tamaulipas & 40.34 & 30.65 & 20.11 & 39.90 & 61.40 & 34.93 & 33.19 & 47.59 & 64.96 & NRD & & & & \\
\hline Tlaxcala & 36.85 & 28.87 & $\mathrm{ENCl}$ & 38.21 & 64.05 & 0.00 & NRA & $\mathrm{ENCl}$ & 56.88 & 95.46 & & & & \\
\hline Veracruz & 38.67 & 30.10 & 13.62 & 46.71 & 65.41 & 42.41 & 18.24 & 51.11 & 64.61 & 92.33 & & & & \\
\hline Yucatán & 41.17 & 37.63 & 19.62 & 42.37 & 66.95 & 0.00 & 52.84 & 52.38 & 60.73 & 94.43 & & & & \\
\hline Zacatecas & 27.95 & 20.66 & 20.83 & 31.12 & 56.39 & 0.00 & 29.23 & $\mathrm{ENCl}$ & 54.63 & 92.82 & & & & \\
\hline
\end{tabular}

* Para total, información de las instituciones públicas y privadas de salud. Fuente: Boletín de Información Estadística 2002;III(22). Programas sustantivos. Dirección General de Información y Evaluación del Desempeño, Secretaría de Salud, México

₹ No se dispone de información por institución y entidad federativa. Fuente: Monitoreo de los indicadores de la Cruzada N acional por la Calidad de los Servicios de Salud. Dirección General de Calidad y Educación en salud, Subsecretaría de Innovación y Calidad, Secretaría de Salud. México

N P: no participó en la medición de los indicadores de la Cruzada N acional por la Calidad de los Servicios de Salud NRD: no reportó datos dentro de la Cruzada N acional por la Calidad de los Servicios de Salud 


\section{Cuadro VII.2 \\ INDICADORES DE RESULTADO, CALIDAD TÉCNICA E INTERPERSONAL, SEGÚN ENTIDAD FEDERATIVA E INSTITUCIÓN. MÉxico, 2002}

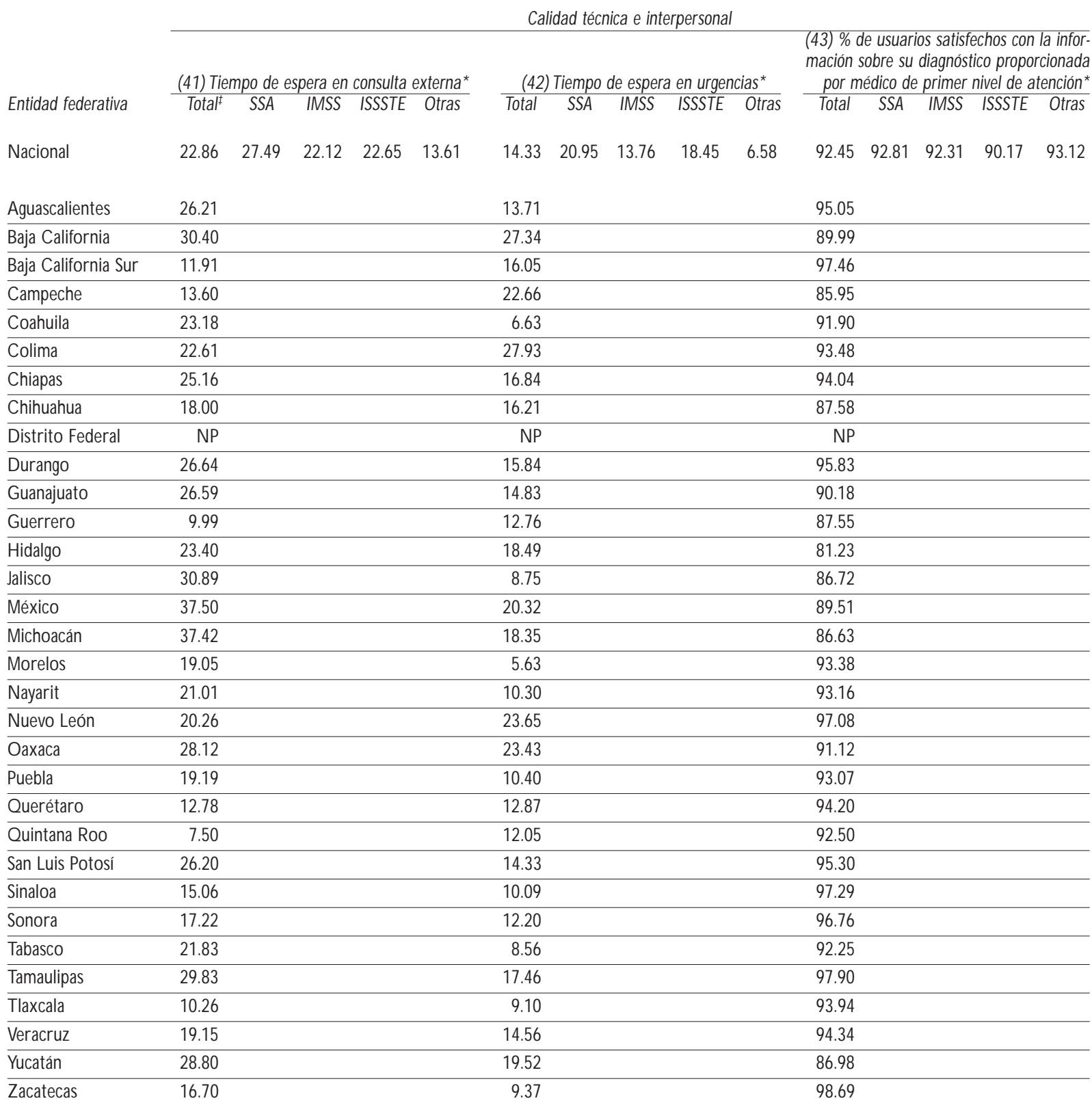

* No se dispone de información por institución y entidad federativa

N P: no participó en la medición de los indicadores de la Cruzada N acional por la Calidad de los Servicios de Salud

Fuente: Monitoreo de los indicadores de la C ruzada N acional por la Calidad de los Servicios de Salud. Dirección General de Calidad y Educación en Salud, Subsecretaría de Innovación y Calidad, Secretaría de Salud, México 


\section{Cuadro VIII.1 \\ INDICADORES DE RESULTADO, EFICIENCIA, SEGÚN ENTIDAD FEDERATIVA E INSTITUCIÓN. MÉxico, 2002}

\begin{tabular}{|c|c|c|c|c|c|c|c|c|c|c|c|c|c|c|c|c|}
\hline \multirow[b]{3}{*}{ Entidad federativa } & \multicolumn{16}{|c|}{ Eficiencia } \\
\hline & \multicolumn{8}{|c|}{$\begin{array}{l}\text { (45) Promedio diario de consultas por médico } \\
\text { de primer nivel de atención en contacto con el paciente }\end{array}$} & \multicolumn{8}{|c|}{$\begin{array}{l}\text { (46) Promedio diario de intervenciones } \\
\text { quirúrgicas por quirófano }{ }^{\ddagger}\end{array}$} \\
\hline & Total & SSA & IMSS-SO| & IMSS & ISSSTE & Pemex* & Sedena & Secmar & Total & SSA & IMSS-Sol & IMSS & ISSSTE & Pemex* & Sedena & Secmar \\
\hline$N$ acional & 13.01 & 9.96 & 16.85 & 17.36 & 12.89 & 9.80 & 8.63 & 11.48 & 2.90 & 2.41 & 3.86 & 3.93 & 2.24 & 1.79 & 0.81 & 0.71 \\
\hline A guascalientes & 12.70 & 7.00 & $\mathrm{ENCl}$ & 20.40 & 14.51 & 0.00 & 6.05 & $\mathrm{ENCl}$ & 3.26 & 2.37 & $\mathrm{ENCl}$ & 5.19 & 2.48 & 0.00 & NDR & $\mathrm{ENCl}$ \\
\hline Baja C alifornia & 2.53 & 9.03 & 14.21 & 17.70 & 7.30 & 0.00 & 5.17 & 8.45 & 2.78 & 4.32 & 3.59 & 3.13 & 1.53 & 0.00 & 0.60 & 0.70 \\
\hline Baja California Sur & 10.19 & 6.03 & $\mathrm{ENCl}$ & 12.51 & 17.93 & 0.00 & 9.56 & 7.38 & 2.08 & 1.90 & $\mathrm{ENCl}$ & 3.58 & 1.34 & 0.00 & 0.51 & 0.21 \\
\hline Campeche & 9.49 & 8.12 & 21.80 & 16.98 & 15.43 & 1.18 & 11.21 & 14.24 & 2.59 & 2.91 & 2.50 & 3.62 & 1.53 & 2.26 & NDR & 0.23 \\
\hline Coahuila & 15.65 & 8.61 & 15.38 & 19.56 & 16.28 & 0.00 & 6.92 & $\mathrm{ENCl}$ & 3.25 & 1.95 & 3.17 & 4.21 & 1.72 & 0.00 & 0.34 & $\mathrm{ENCl}$ \\
\hline Colima & 10.21 & 6.75 & $\mathrm{ENCl}$ & 18.60 & 11.73 & 0.00 & 8.47 & 9.39 & 4.14 & 3.40 & $\mathrm{ENCl}$ & 7.49 & 2.28 & 0.00 & NDR & 2.27 \\
\hline Chiapas & 12.75 & 12.19 & 15.44 & 14.70 & 12.51 & 8.28 & 12.97 & 21.45 & 2.68 & 2.77 & 3.68 & 3.62 & 1.74 & NDR & 1.02 & 0.85 \\
\hline Chihuahua & 13.85 & 9.16 & 15.02 & 17.38 & 12.54 & 26.62 & 12.05 & $\mathrm{ENCl}$ & 2.91 & 1.71 & 1.80 & 4.62 & 2.00 & NDR & 0.53 & $\mathrm{ENCl}$ \\
\hline Distrito Federal & 11.20 & 7.50 & 29.88 & 15.51 & 9.92 & 8.54 & 6.06 & 41.88 & 2.15 & 1.82 & NDR & 2.89 & 2.01 & 1.29 & 1.09 & 1.30 \\
\hline Durango & 12.06 & 7.02 & 17.79 & 15.47 & 15.16 & 0.00 & 7.45 & $\mathrm{ENCl}$ & 3.53 & 2.30 & 5.34 & 4.96 & 3.00 & 0.00 & 0.33 & $\mathrm{ENCl}$ \\
\hline Guanajuato & 12.43 & 9.15 & $\mathrm{ENCl}$ & 18.85 & 13.32 & 9.03 & 16.57 & $\mathrm{ENCl}$ & 4.32 & 4.92 & $\mathrm{ENCl}$ & 4.81 & 2.57 & 1.89 & 0.89 & $\mathrm{ENCl}$ \\
\hline Guerrero & 11.02 & 10.10 & $\mathrm{ENCl}$ & 15.65 & 13.36 & 0.00 & 11.25 & 3.21 & 3.53 & 3.15 & $\mathrm{ENCl}$ & 7.10 & 2.26 & 0.00 & 1.00 & 0.56 \\
\hline Hidalgo & 13.22 & 11.03 & 16.33 & 17.82 & 19.50 & 6.71 & 12.69 & $\mathrm{ENCl}$ & 3.55 & 3.40 & 4.71 & 4.15 & 2.22 & 1.82 & NDR & $\mathrm{ENCl}$ \\
\hline Jalisco & 11.97 & 9.07 & $\mathrm{ENCl}$ & 15.37 & 15.64 & 7.66 & 5.94 & 11.32 & 2.34 & 1.24 & $\mathrm{ENCl}$ & 3.44 & 1.76 & NDR & 0.69 & 0.23 \\
\hline México & 16.19 & 16.42 & $\mathrm{ENCl}$ & 15.99 & 15.87 & 13.41 & 13.94 & $\mathrm{ENCl}$ & 3.35 & 3.08 & $\mathrm{ENCl}$ & 4.33 & 1.36 & NDR & 0.39 & $\mathrm{ENCl}$ \\
\hline Michoacán & 13.83 & 10.67 & 17.23 & 21.61 & 12.38 & 0.00 & 11.80 & 7.01 & 2.62 & 2.32 & 4.25 & 3.46 & 1.83 & 0.00 & 0.26 & 0.37 \\
\hline Morelos & 13.48 & 10.07 & $\mathrm{ENCl}$ & 21.18 & 14.78 & 0.00 & 13.65 & $\mathrm{ENCl}$ & 3.81 & 3.85 & $\mathrm{ENCl}$ & 4.27 & 2.69 & 0.00 & 0.83 & $\mathrm{ENCl}$ \\
\hline $\mathrm{N}$ ayarit & 12.26 & 8.16 & 12.79 & 24.62 & 13.85 & 0.00 & 7.09 & 13.44 & 3.66 & 3.10 & 4.92 & 3.70 & 4.64 & 0.00 & NDR & NDR \\
\hline N uevo León & 15.89 & 9.98 & $\mathrm{ENCl}$ & 22.29 & 9.82 & 10.47 & 24.02 & $\mathrm{ENCl}$ & 4.39 & 2.43 & $\mathrm{ENCl}$ & 5.39 & 3.19 & 2.24 & 0.60 & $\mathrm{ENCl}$ \\
\hline 0 axaca & 11.97 & 10.49 & 12.14 & 16.66 & 16.60 & 10.77 & 14.23 & 4.55 & 2.86 & 2.93 & 3.24 & 3.77 & 2.40 & 2.26 & 0.54 & 0.63 \\
\hline Puebla & 13.12 & 10.29 & 19.39 & 16.68 & 9.42 & 18.00 & 14.60 & $\mathrm{ENCl}$ & 2.52 & 1.25 & 4.58 & 3.81 & 1.76 & 0.25 & 0.98 & $\mathrm{ENCl}$ \\
\hline Q uerétaro & 13.46 & 10.92 & $\mathrm{ENCl}$ & 19.04 & 13.27 & 0.00 & 22.50 & $\mathrm{ENCl}$ & 5.84 & 4.56 & $\mathrm{ENCl}$ & 5.70 & 11.45 & 0.00 & NDR & $\mathrm{ENCl}$ \\
\hline Q uintana Roo & 11.70 & 7.87 & $\mathrm{ENCl}$ & 19.24 & 18.55 & $\mathrm{ENCl}$ & 9.72 & 10.27 & 3.24 & 2.41 & $\mathrm{ENCl}$ & 5.97 & 1.76 & $\mathrm{ENCl}$ & 0.35 & 0.19 \\
\hline San Luis Potosí & 13.88 & 9.49 & 17.59 & 17.74 & 15.28 & 4.86 & 21.87 & $\mathrm{ENCl}$ & 4.06 & 3.52 & 5.79 & 6.60 & 1.92 & 0.38 & 0.39 & $\mathrm{ENCl}$ \\
\hline Sinaloa & 14.66 & 9.29 & 17.86 & 18.68 & 15.21 & 47.12 & 21.96 & 9.56 & 2.66 & 2.45 & 1.96 & 3.11 & 3.59 & $N D R$ & 0.27 & 0.32 \\
\hline Sonora & 14.64 & 8.38 & $\mathrm{ENCl}$ & 19.31 & 14.32 & 0.00 & 9.43 & 3.60 & 2.46 & 2.03 & $\mathrm{ENCl}$ & 3.46 & 1.55 & 0.00 & 1.30 & 0.09 \\
\hline Tabasco & 12.34 & 10.56 & $\mathrm{ENCl}$ & 14.37 & 13.00 & 11.68 & 9.97 & 16.98 & 3.23 & 3.66 & $\mathrm{ENCl}$ & 4.24 & 1.85 & 2.00 & 0.75 & 0.32 \\
\hline Tamaulipas & 11.79 & 6.51 & 17.11 & 16.72 & 14.89 & 15.54 & 9.55 & 4.45 & 3.24 & 3.35 & 3.37 & 4.46 & 2.18 & 2.65 & 0.35 & 0.85 \\
\hline Tlaxcala & 10.08 & 8.75 & $\mathrm{ENCl}$ & 15.01 & 12.30 & 0.00 & 5.43 & $\mathrm{ENCl}$ & 3.74 & 3.16 & $\mathrm{ENCl}$ & 6.30 & 2.08 & 0.00 & NDR & $\mathrm{ENCl}$ \\
\hline Veracruz & 12.08 & 8.56 & 18.02 & 15.76 & 13.63 & 11.52 & 17.05 & 12.60 & 2.80 & 2.17 & 5.00 & 3.89 & 2.83 & 1.87 & 0.97 & 1.50 \\
\hline Yucatán & 20.73 & 12.24 & 36.74 & 31.22 & 11.75 & 0.00 & 11.89 & 24.24 & 3.26 & 2.94 & 3.31 & 3.85 & 3.15 & 0.00 & 1.13 & 0.10 \\
\hline Zacatecas & 12.39 & 10.80 & 13.51 & 13.48 & 15.11 & 0.00 & 18.85 & $\mathrm{ENCl}$ & 3.14 & 2.72 & 3.38 & 4.11 & 2.77 & 0.00 & 0.58 & $\mathrm{ENCl}$ \\
\hline
\end{tabular}

EN Cl: entidad no cubierta por la institución

NDR: no dispone de este tipo de recursos

Fuente: Boletín de Información Estadística 2002;1(22). Recursos y Servicios. Dirección General de Información y Evaluación del D esempeño, Secretaría de Salud, México 


\section{Cuadro VIII.2 \\ INDICADORES DE RESULTADO, EFICIENCIA, SEGÚN ENTIDAD FEDERATIVA E INSTITUCIÓN. MÉXICO, 2002}

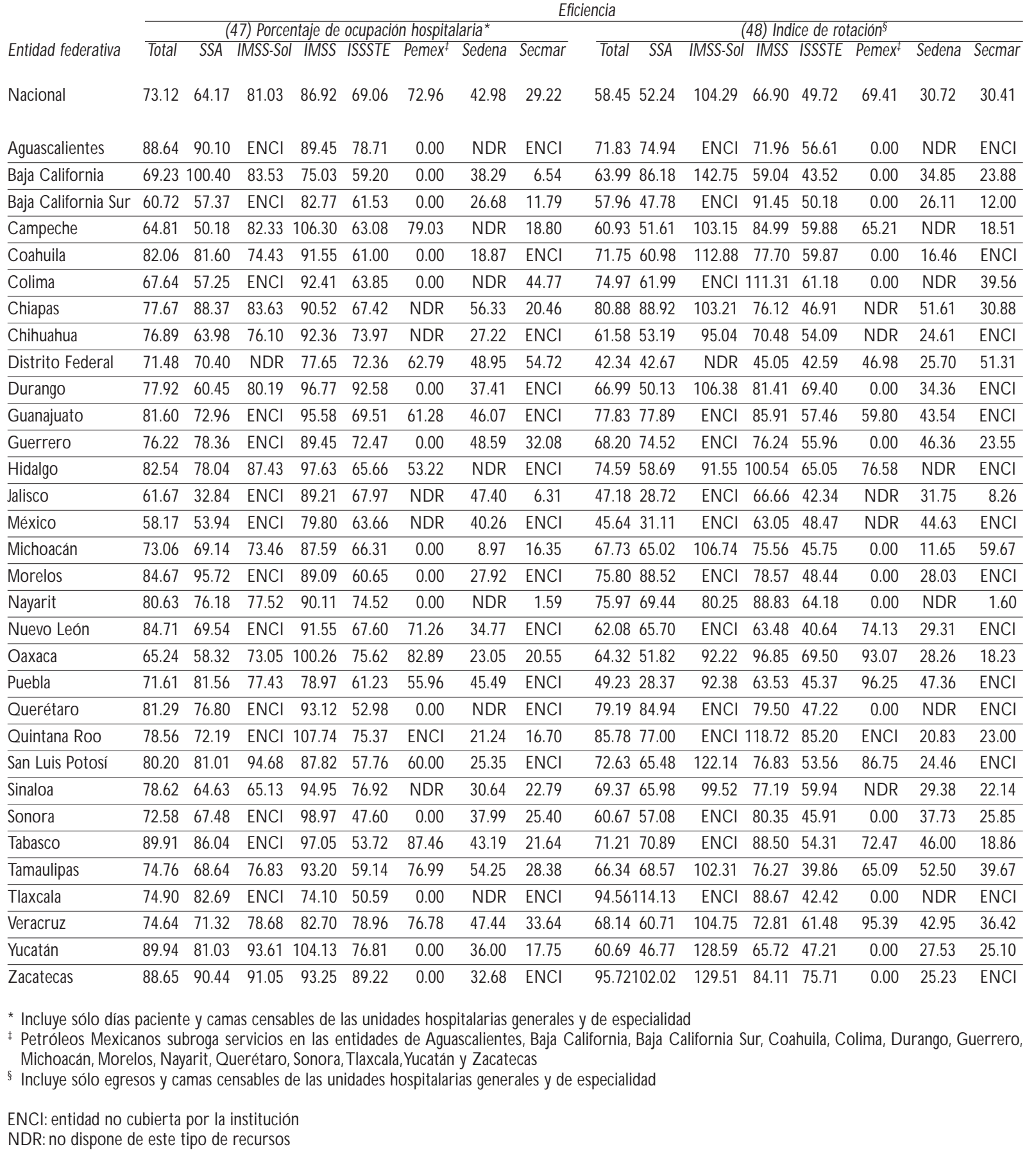

Fuente: Boletín de Información Estadística 2002;1(22). Recursos y Servicios. Dirección General de Información y Evaluación del D esempeño, Secretaría de Salud, México 


\section{Cuadro VIII.3 INDICADORES DE RESULTADo, EFICIENCIA, POR ENTIDAD FEDERATIVA, INSTITUCIÓN. MÉXICo 2002}

Eficiencia

\begin{tabular}{|c|c|c|c|c|}
\hline \multicolumn{5}{|c|}{ (49) Distribución del presupuesto ejercido según rubro en relación con el total de presupuesto ejercido } \\
\hline Personal (capítulo 1000$)$ & $\begin{array}{l}\text { Medicamentos y materiales } \\
\text { de curación (concepto } 2500 \text { ) }\end{array}$ & $\begin{array}{l}\text { Conservación y mantenimiento } \\
\text { (concepto } 3 \text { 500) }\end{array}$ & & Resto \\
\hline Población Población asegurada & Población Población asegurada & Población Población asegurada & Población & Población asegurada \\
\hline
\end{tabular}
Entidad federativa no asegurada IMSS ISSSTE Pemex no asegurada IMSS ISSSTE Pemex no asegurada IMSS ISSSTE Pemex no asegurada IMSS ISSSTE Pemex

\begin{tabular}{|c|c|c|c|c|c|c|c|c|c|c|c|c|c|c|c|c|}
\hline N acional & 64.09 & 71.67 & 65.87 & 58.79 & 3.39 & 18.68 & 19.19 & 26.65 & 0.94 & 0.27 & 3.91 & 0.00 & 31.59 & 9.38 & 11.03 & 14.56 \\
\hline Aguascalientes & 73.07 & 68.67 & 79.73 & 7.22 & 4.09 & 22.08 & 2.94 & 30.65 & 0.84 & 0.61 & 3.00 & 0.00 & 22.00 & 8.65 & 14.32 & 62.12 \\
\hline Baja California & 75.27 & 73.29 & 80.19 & 13.48 & 3.66 & 16.47 & 2.95 & 34.77 & 0.01 & 0.31 & 3.25 & 0.00 & 21.06 & 9.94 & 13.61 & 51.75 \\
\hline Baja California Sur & 77.74 & 74.95 & 80.53 & 20.47 & 4.56 & 11.97 & 1.74 & 33.56 & 0.65 & 0.54 & 2.00 & 0.00 & 17.05 & 12.54 & 15.74 & 45.97 \\
\hline Campeche & 71.92 & 68.57 & 70.08 & 69.83 & 6.37 & 13.63 & 3.42 & 20.10 & 1.64 & 0.97 & 9.33 & 0.00 & 20.08 & 16.84 & 17.17 & 10.07 \\
\hline Coahuila & 73.39 & 71.23 & 83.81 & 16.36 & 2.23 & 19.19 & 1.89 & 37.02 & 0.47 & 0.35 & 2.41 & 0.00 & 23.91 & 9.23 & 11.89 & 46.62 \\
\hline Colima & 75.69 & 74.79 & 83.12 & 3.84 & 0.14 & 15.01 & 3.08 & 48.09 & 0.51 & 0.27 & 2.75 & 0.00 & 23.66 & 9.92 & 11.04 & 8.07 \\
\hline Chiapas & 71.27 & 73.48 & 79.87 & 67.10 & 4.64 & 12.43 & 2.89 & 23.70 & 1.31 & 0.72 & 1.80 & 0.00 & 22.79 & 13.36 & 15.43 & 9.20 \\
\hline Chihuahua & 73.10 & 73.49 & 81.04 & 21.47 & 1.94 & 17.65 & 2.38 & 25.38 & 0.86 & 0.17 & 3.63 & 0.00 & 24.11 & 8.69 & 12.94 & 53.15 \\
\hline Distrito Federal & 60.55 & 69.26 & 49.26 & 61.01 & 2.90 & 18.41 & 37.46 & 21.69 & 1.00 & 0.08 & 5.04 & 0.00 & 35.54 & 12.25 & 8.24 & 17.30 \\
\hline Durango & 77.13 & 74.13 & 81.41 & 11.71 & 3.06 & 17.69 & 5.39 & 25.65 & 0.78 & 0.29 & 2.24 & 0.00 & 19.03 & 7.90 & 10.96 & 62.64 \\
\hline Guanajuato & 68.05 & 69.56 & 80.44 & 61.78 & 5.03 & 22.62 & 3.12 & 27.98 & 1.85 & 0.10 & 4.57 & 0.00 & 25.07 & 7.72 & 11.87 & 10.24 \\
\hline Guerrero & 74.67 & 74.77 & 81.75 & 6.51 & 3.17 & 14.15 & 1.69 & 46.34 & 0.82 & 0.46 & 3.17 & 0.00 & 21.34 & 10.62 & 13.39 & 47.15 \\
\hline Hidalgo & 73.96 & 75.50 & 84.52 & 75.59 & 2.61 & 15.74 & 2.37 & 18.14 & 0.21 & 0.13 & 2.76 & 0.00 & 23.22 & 8.63 & 10.35 & \\
\hline Jalisco & 76.36 & 72.47 & 80.86 & 40.78 & 3.06 & 19.54 & 3.99 & 26.47 & 1.04 & 0.38 & 2.50 & 0.00 & 19.53 & 7.62 & 12.66 & 32.74 \\
\hline México & 66.57 & 76.61 & 76.76 & 40.04 & 3.07 & 17.60 & 11.62 & 40.15 & 0.20 & 0.19 & 1.14 & 0.00 & 30.16 & 5.60 & 10.47 & 19.81 \\
\hline Michoacán & 71.66 & 72.30 & 83.96 & 6.50 & 3.11 & 18.26 & 2.05 & 37.39 & 0.98 & 0.56 & 2.50 & 0.00 & 24.24 & 8.88 & 11.49 & 56.11 \\
\hline Morelos & 76.37 & 75.70 & 83.31 & 8.89 & 1.31 & 15.88 & 2.12 & 47.78 & 0.81 & 0.06 & 2.77 & 0.00 & 21.51 & 8.36 & 11.80 & 43.32 \\
\hline $\bar{N}$ ayarit & 76.34 & 75.44 & 82.31 & 9.91 & 4.33 & 15.38 & 1.87 & 45.92 & 1.41 & 0.18 & 2.56 & 0.00 & 17.91 & 9.01 & 13.27 & 44.17 \\
\hline Nuevo León & 71.24 & 68.38 & 79.89 & 53.85 & 2.66 & 23.69 & 3.49 & 22.34 & 0.61 & 0.38 & 3.45 & 0.00 & 25.49 & 7.55 & 13.18 & 23.81 \\
\hline 0 axaca & 70.48 & 74.08 & 74.39 & 75.05 & 1.05 & 15.60 & 4.81 & 18.69 & 0.46 & 0.48 & 2.98 & 0.00 & 28.01 & 9.84 & 17.83 & 6.25 \\
\hline Puebla & 69.22 & 69.64 & 82.12 & 45.00 & 3.46 & 22.61 & 3.70 & 31.57 & 2.27 & 0.37 & 2.49 & 0.00 & 25.06 & 7.38 & 11.69 & 23.42 \\
\hline Q uerétaro & 74.12 & 72.12 & 84.81 & 9.71 & 3.78 & 18.99 & 1.93 & 41.10 & 0.59 & 0.28 & 2.18 & 0.00 & 21.51 & 8.62 & 11.08 & 49.19 \\
\hline Q uintana Roo & 78.45 & 77.09 & 74.87 & 0.00 & 2.56 & 13.06 & 6.11 & 0.00 & 0.69 & 0.18 & 3.59 & 0.00 & 18.30 & 9.67 & 15.44 & 100.00 \\
\hline San Luis Potosí & 69.22 & 67.85 & 78.15 & 45.18 & 5.31 & 22.71 & 2.22 & 31.67 & 0.79 & 0.18 & 2.63 & 0.00 & 24.68 & 9.26 & 17.00 & 23.15 \\
\hline Sinaloa & 71.79 & 68.39 & 71.41 & 16.97 & 2.56 & 19.70 & 8.25 & 32.02 & 0.70 & 0.25 & 0.85 & 0.00 & 24.96 & 11.66 & 19.49 & 51.02 \\
\hline Sonora & 68.61 & 65.98 & 81.04 & 9.38 & 7.20 & 20.86 & 1.56 & 35.43 & 1.29 & 0.58 & 5.06 & 0.00 & 22.91 & 12.57 & 12.34 & 55.19 \\
\hline Tabasco & 74.46 & 78.30 & 74.42 & 61.41 & 5.55 & 13.26 & 0.99 & 30.34 & 0.41 & 0.89 & 6.29 & 0.00 & 19.58 & 7.55 & 18.30 & 8.25 \\
\hline Tamaulipas & 76.80 & 73.43 & 86.05 & 56.58 & 1.22 & 18.40 & 1.60 & 32.87 & 0.29 & 0.05 & 1.11 & 0.00 & 21.68 & 8.12 & 11.25 & 10.55 \\
\hline Tlaxcala & 74.62 & 79.86 & 86.08 & 0.00 & 6.79 & 13.95 & 1.56 & 50.70 & 1.09 & 0.54 & 13.05 & 0.00 & 17.50 & 5.64 & -0.68 & 49.30 \\
\hline Veracruz & 68.61 & 75.96 & 84.88 & 65.01 & 4.03 & 15.90 & 1.25 & 26.22 & 2.11 & 0.32 & 2.38 & 0.00 & 25.25 & 7.82 & 11.49 & 8.77 \\
\hline Yucatán & 70.61 & 68.21 & 66.34 & 8.44 & 3.65 & 20.56 & 6.94 & 44.23 & 1.07 & 0.51 & 4.51 & 0.00 & 24.67 & 10.73 & 22.21 & 47.33 \\
\hline Zacatecas & 73.36 & 72.43 & 83.27 & 13.28 & 2.34 & 18.73 & 3.16 & 37.34 & 0.76 & 0.03 & 2.08 & 0.00 & 23.54 & 8.81 & 11.49 & \\
\hline
\end{tabular}

Notas:

1. El presupuesto total para población no asegurada incluye Ramo 12, Ramo 33 y Gasto Estatal. El Ramo 12 incluye Programas Especiales, Unidades Centrales, 0 rganismos D escentralizados y 0 rganos D esconcentrados; la distribución se realizó de la siguiente manera: las transferencias directas se asignaron por entidad federativa en lo que se refiere a programas como PAC, 0 portunidades, IMSS-O portunidades, Seguro Popular, Comunidades Saludables y Cruzada N acional. Lo correspondiente a Unidades Centrales se considera como gasto no distribuible. El Hospital Manuel Gea González, el Hospital General de México, el Hospital Infantil, el Hospital Juárez de México y los Institutos N acionales en función de los egresos hospitalarios. Por último, el resto de 0 rganismos D escentralizados y 0 rganos D esconcentrados se considera de igual manera gasto no distribuible

2. El resto incluye los Capítulos: 2000 (Materiales y Suministros, excepto partida 2504 y 2 505), 3000 (Servicios G enerales, excepto concepto 3500 ), 4000 (Transferencias), 5000 (Bienes Muebles e Inmuebles), 6000 (0 bras Públicas) y 7000 (Inversión Financiera, Provisiones Económicas, Ayudas, 0 tras Erogaciones y Pensiones y 0 tras)

3. El presupuesto total para población asegurada incluye Instituto Mexicano del Seguro Social, Instituto de Seguridad y Servicios Sociales de los Trabajadores del Estado y Petróleos Mexicanos

4. Para conformar el gasto en administración se incluyeron las erogaciones de los conceptos 11, 13 (de los cuales sólo se tomó el porcentaje de recursos humanos destinados a administración por entidad e institución), 16 y 17 del Capítulo 1 000, las actividades relacionadas con la compra de materiales y útiles de administración y enseñanza, herramientas, refacciones y accesorios, materiales y artículos de construcción, combustible, lubricantes y aditivos, así como el pago de servicios básicos de arrendamiento, asesoría, consultoría, informáticos, estudios e investigaciones, de mantenimiento y conservación, impresión, publicación, difusión e información y servicios oficiales

Fuente: Boletín de Información Estadística 2002;IIIV(22). Dirección General de Información y Evaluación del Desempeño, y Dirección General de Planeación y Desarrollo en Salud, Secretaría de Salud, México 


\section{Cuadro VIII.4 \\ INDICADORES DE RESULTADO, EFICIENCIA, SEGÚN ENTIDAD FEDERATIVA E INSTITUCIÓN. MÉXICO, 2002}

\begin{tabular}{|c|c|c|c|c|c|c|c|c|}
\hline \multirow{3}{*}{ Entidad federativa } & \multicolumn{8}{|c|}{ Eficiencia } \\
\hline & \multicolumn{8}{|c|}{ (51) Prestadores de servicios clínicos como porcentaje del total de trabajadores } \\
\hline & Total & SSA & IMSS-Sol & IMSS & ISSSTE & Pemex* & Sedena & Secmar \\
\hline Nacional & 66.99 & 70.81 & 73.74 & 61.73 & 71.59 & 59.96 & 93.35 & 69.95 \\
\hline Aguascalientes & 68.08 & 71.81 & $\mathrm{ENCl}$ & 62.47 & 74.27 & 0.00 & 100.00 & $\mathrm{ENCl}$ \\
\hline Baja California & 65.37 & 73.83 & 65.71 & 61.42 & 73.09 & 0.00 & 95.28 & 40.82 \\
\hline Baja C alifornia Sur & 65.75 & 66.31 & $\mathrm{ENCl}$ & 61.72 & 69.86 & 0.00 & 89.60 & 70.13 \\
\hline Campeche & 67.87 & 69.10 & 71.76 & 62.46 & 72.87 & 69.60 & 100.00 & 68.71 \\
\hline Coahuila & 66.08 & 74.28 & 71.63 & 61.96 & 70.21 & 0.00 & 92.12 & $\mathrm{ENCl}$ \\
\hline Colima & 68.59 & 75.55 & $\mathrm{ENCl}$ & 60.55 & 71.34 & 0.00 & 100.00 & 65.36 \\
\hline Chiapas & 73.82 & 81.53 & 73.95 & 59.55 & 70.74 & 56.12 & 97.16 & 92.75 \\
\hline Chihuahua & 65.94 & 73.19 & 74.06 & 60.53 & 70.80 & 57.69 & 94.12 & $\mathrm{ENCl}$ \\
\hline Distrito Federal & 64.79 & 62.82 & 2.38 & 61.99 & 73.74 & 58.66 & 90.73 & 97.28 \\
\hline Durango & 68.69 & 72.16 & 75.06 & 61.55 & 75.95 & 0.00 & 99.23 & $\mathrm{ENCl}$ \\
\hline Guanajuato & 69.17 & 76.96 & $\mathrm{ENCl}$ & 63.98 & 67.30 & 58.38 & 94.46 & $\mathrm{ENCl}$ \\
\hline Guerrero & 72.80 & 77.94 & $\mathrm{ENCl}$ & 59.64 & 73.73 & 0.00 & 94.94 & 70.22 \\
\hline Hidalgo & 70.29 & 76.07 & 74.38 & 60.58 & 67.66 & 57.24 & 100.00 & $\mathrm{ENCl}$ \\
\hline Jalisco & 68.43 & 72.28 & $\mathrm{ENCl}$ & 64.40 & 74.96 & 61.82 & 92.90 & 62.22 \\
\hline México & 65.92 & 69.48 & $\mathrm{ENCl}$ & 61.55 & 65.45 & 73.26 & 97.27 & $\mathrm{ENCl}$ \\
\hline Michoacán & 67.15 & 69.55 & 77.64 & 59.90 & 68.04 & 0.00 & 100.00 & 60.40 \\
\hline Morelos & 68.67 & 76.52 & $\mathrm{ENCl}$ & 60.23 & 69.01 & 0.00 & 96.06 & $\mathrm{ENCl}$ \\
\hline $\mathrm{N}$ ayarit & 68.30 & 79.12 & 77.92 & 56.38 & 72.45 & 0.00 & 100.00 & 41.18 \\
\hline Nuevo León & 66.04 & 74.61 & $\mathrm{ENCl}$ & 62.61 & 73.15 & 58.38 & 93.98 & $\mathrm{ENCl}$ \\
\hline 0 axaca & 70.98 & 75.60 & 74.15 & 59.17 & 70.38 & 58.95 & 95.37 & 51.75 \\
\hline Puebla & 67.72 & 75.20 & 74.85 & 61.84 & 69.62 & 64.39 & 92.66 & $\mathrm{ENCl}$ \\
\hline Q uerétaro & 69.34 & 75.69 & $\mathrm{ENCl}$ & 62.49 & 72.54 & 0.00 & 100.00 & $\mathrm{ENCl}$ \\
\hline Q uintana Roo & 67.52 & 76.89 & $\mathrm{ENCl}$ & 58.87 & 68.30 & $\mathrm{ENCl}$ & 98.65 & 61.54 \\
\hline San Luis Potosí & 66.59 & 69.18 & 75.00 & 60.28 & 72.30 & 55.56 & 94.49 & $\mathrm{ENCl}$ \\
\hline Sinaloa & 67.01 & 70.26 & 75.05 & 62.31 & 73.34 & 55.56 & 90.43 & 74.10 \\
\hline Sonora & 64.46 & 67.56 & $\mathrm{ENCl}$ & 59.80 & 66.44 & 0.00 & 93.75 & 50.87 \\
\hline Tabasco & 64.78 & 70.10 & $\mathrm{ENCl}$ & 57.07 & 77.35 & 59.66 & 95.15 & 66.67 \\
\hline Tamaulipas & 65.62 & 67.50 & 76.16 & 62.40 & 67.79 & 61.72 & 91.75 & 74.01 \\
\hline Tlaxcala & 69.35 & 76.63 & $\mathrm{ENCl}$ & 59.43 & 69.67 & 0.00 & 100.00 & $\mathrm{ENCl}$ \\
\hline Veracruz & 67.61 & 76.83 & 79.95 & 60.44 & 67.90 & 59.72 & 94.00 & 64.37 \\
\hline Yucatán & 68.86 & 75.79 & 71.21 & 64.13 & 67.15 & 0.00 & 96.93 & 54.22 \\
\hline Zacatecas & 71.96 & 79.57 & 72.14 & 62.33 & 76.41 & 0.00 & 98.36 & $\mathrm{ENCl}$ \\
\hline
\end{tabular}

* Petróleos Mexicanos subroga servicios en las entidades de Aguascalientes, Baja California, Baja California Sur, Coahuila, Colima, Durango, Guerrero, Michoacán, Morelos, N ayarit, Q uerétaro, Sonora,Tlaxcala, Yucatán y Zacatecas

EN Cl: entidad no cubierta por la institución

Fuente: Boletín de Información Estadística 2002;1(22). Recursos y Servicios. Dirección General de Información y Evaluación del D esempeño, Secretaría de Salud, México 